TRANSACTIONS OF THE

AMERICAN MATHEMATICAL SOCIETY

Volume 354, Number 1, Pages 63-74

S 0002-9947(01)02809-4

Article electronically published on April 24, 2001

\title{
CONSTRUCTION DE CERTAINES OPÉRADES ET BIGĖBRES ASSOCIÉES AUX POLYTOPES DE STASHEFF ET HYPERCUBES
}

\author{
FRÉDÉRIC CHAPOTON
}

\begin{abstract}
Stasheff polytopes, introduced by Stasheff in his study of $\mathrm{H}$ spaces, are linked to associativity. The direct sum of their cellular complexes is the underlying complex of the $A_{\infty}$ operad which describes homotopy associative algebras. In particular, there exists a quasi-isomorphism $A_{\infty} \rightarrow$ As.

Here, we define on the direct sum of their dual cellular complexes the structure of a differential graded operad. This construction extends the dendriform operad of Loday, which corresponds to the vertices of the polytopes. We also define the structure of a differential graded operad on the direct sum of the dual cellular complexes of the hypercubes. We define a quasi-isomorphism from As to each of these operads.

We also define non-differential variants of the two preceding operads and a morphism from As to each of these operads. We show that the free algebras have a coproduct which turns them into bialgebras.

RÉsumé. Les polytopes de Stasheff, introduits pour l'étude des $H$-espaces, sont liés à l'associativité. La somme directe de leurs complexes cellulaires forme le complexe sous-jacent à l'opérade $A_{\infty}$ qui décrit les algèbres associatives à homotopie près. En particulier, il existe un quasi-isomorphisme $A_{\infty} \rightarrow$ As.

Ici, on munit la somme directe des duaux de leurs complexes cellulaires d'une structure d'opérade différentielle graduée. Cette construction généralise l'opérade des algèbres dendriformes de Loday, qui correspond aux sommets des polytopes. On munit aussi la somme directe des duaux des complexes cellulaires des hypercubes d'une structure d'opérade différentielle graduée. On définit un quasi-isomorphisme de As dans chacune de ces deux opérades.

On construit également des variantes non différentielles des deux opérades précédentes. On définit un morphisme de As dans chacune de ces opérades et on montre que les algèbres libres sont munies d'un coproduit coassociatif qui en fait des bigèbres.
\end{abstract}

\section{INTRODUCTION}

Les polytopes de Stasheff sont des objets classiques, apparus en liaison avec l'opérade As qui décrit les algèbres associatives, plus précisément dans l'étude par Stasheff des multiplications sur les espaces topologiques [Sta63]. La somme directe des complexes cellulaires de ces polytopes intervient aussi comme complexe sousjacent à l'opérade différentielle graduée $A_{\infty}$ qui décrit les algèbres associatives à homotopie près.

Received by the editors April 21, 2000.

2000 Mathematics Subject Classification. Primary 18D50, 52B11; Secondary 16W30.

Key words and phrases. Operads, polytopes, permutohedra, associahedra, bialgebras. 
On les considère ici d'un point de vue qui semble distinct de leur relation avec l'associativité. Une opérade Dend des algèbres dendriformes, qui fait intervenir les arbres binaires plans, a été définie par Jean-Louis Loday dans [Lod99. Par la suite, Loday et Ronco ont montré dans [LR98, Lod99, Ron00] que les Dend-algèbres libres sont des bigèbres. En particulier, l'algèbre dendriforme libre sur un générateur est une bigèbre qui admet une base indexée par les arbres binaires plans, qu'on peut voir comme les sommets des polytopes de Stasheff. Dans [Cha00a, Cha00b], on a construit diverses bigèbres qui toutes admettent une base indexée par tous les arbres plans, c'est-à-dire par toutes les faces des polytopes de Stasheff. Ces bigèbres admettent pour quotient la bigèbre des arbres binaires plans de Loday et Ronco. Par analogie avec le cas des arbres binaires plans et de l'opérade dendriforme, on peut alors se demander si ces bigèbres sont des algèbres libres sur certaines opérades. Cet article répond positivement à cette question, en construisant les opérades attendues. On obtient ici trois extensions distinctes de l'opérade dendriforme, qui correspondent aux différentes bigèbres construites dans Cha00a, Cha00b] .

Dans [LR98, Loday et Ronco montrent par ailleurs que la bigèbre des arbres binaires plans contient la bigèbre des descentes de Solomon introduite par Malvenuto et Reutenauer dans [MR95], nommée aussi bigèbre des fonctions symétriques noncommutatives. Cette bigèbre admet une base qu'il est tentant d'indexer par les sommets des hypercubes. Dans Cha00a, Cha00b], on a défini des bigèbres ayant des bases indexées par toutes les faces des hypercubes. Ces bigèbres admettent pour quotient la bigèbre des descentes. On obtient ici des résultats similaires à ceux obtenus pour les polytopes de Stasheff, c.-à-d. on interprète ces bigèbres sur les faces des hypercubes comme des algèbres libres à un générateur pour certaines opérades.

Comme bénéfice de cette reformulation en termes d'opérades, on obtient des familles de bigèbres formées par les algèbres libres sur chacune de ces opérades, le cas des algèbres libres à un générateur correspondant aux résultats de Cha00a Cha00b.

Le plan de l'article est le suivant :

La première section concerne les opérades sur les faces des polytopes de Stasheff. Après des rappels sur les arbres plans et les arbres décorés, on y définit trois opérades, un morphisme de As dans chacune et on montre que les algèbres libres sont des bigèbres.

Dans la seconde section, on obtient des résultats similaires pour des opérades sur les faces des hypercubes.

\section{Opérades sur les faces des polytopes de Stasheff}

1.1. Généralités sur les arbres plans. Soient $n \geq 1$ et $0 \leq d \leq n-1$, on note $\mathrm{AP}^{d}(n)$ l'ensemble des arbres plans à $n+1$ feuilles et $n-d$ sommets internes, chaque arête étant orientée et chaque sommet interne ayant au moins deux arêtes entrantes et exactement une arête sortante. On appelle racine l'unique arête qui n'est pas entrante dans un sommet interne. On considère les arêtes comme des segments ouverts. L'entier $n$ est appelé l'ordre de $T$, noté ord $(T)$, et $d$ est appelé la dimension de l'arbre $T$, notée $\operatorname{dim}(T)$. On note $\operatorname{Som}(T)$ l'ensemble des sommets internes. On représente les arbres les feuilles en haut et la racine en bas. Cette convention étant fixée, on peut alors parler de droite et de gauche. 
On appelle secteur d'un sommet interne $s \in \operatorname{Som}(T)$ un couple d'arêtes entrantes en $s$ consécutives. Soit $\operatorname{Sect}(s)$ l'ensemble des secteurs de $s$ et $\operatorname{Sect}(T)=\bigsqcup_{s} \operatorname{Sect}(s)$ l'ensemble des secteurs de $T$. Il y a une bijection naturelle entre $\operatorname{Sect}(T)$ et l'ensemble des couples de feuilles consécutives, donc le cardinal de $\operatorname{Sect}(T)$ est $\operatorname{ord}(T)$. Moins formellement, on considère aussi un secteur comme une "zone" de l'arbre. On note $\operatorname{ord}(s)$ le cardinal de $\operatorname{Sect}(s)$. Si $v \in \operatorname{Sect}(T)$, on appelle sommet de $v$ l'unique sommet $s(v) \in \operatorname{Som}(T)$ tel que $v \in \operatorname{Sect}(s)$.

On aura besoin par la suite de la notion d'arbre décoré.

Définition 1. Soit $\Gamma$ un ensemble fini. On appelle arbre décoré par $\Gamma$ un couple $(T, \gamma)$, où $T$ est un arbre plan et $\gamma$ une application $\operatorname{de} \operatorname{Sect}(T)$ dans $\Gamma$.

A la différence des arbres décorés utilisés par exemple par Connes et Kreimer [CK98, on décore ici les secteurs et non pas les sommets.

1.2. Une opérade de groupes abéliens gradués. On va définir une opérade $\mathcal{A} \mathcal{P}^{\bullet}$ dans la catégorie monoïdale symétrique $\mathrm{Ab}_{g r}$ des groupes abéliens $\mathbb{Z}$-gradués, munie du produit tensoriel usuel et de la volte $\tau$ définie par $\tau(u \otimes v)=v \otimes u$.

La forme particulière de la composition a été inspirée par la description par Loday de la composition de l'opérade Dend, voir [Lod99 Prop. 5.11]. La notion clé de secteur, qui permet de reformuler la composition de Dend, semble nouvelle. On peut toutefois la rapprocher de la notion d' "angle" introduite par Kontsevich [KS00, p. 29] pour un usage différent. En utilisant la notion de secteur, on peut étendre le principe de la composition de Dend des arbres binaires plans à tous les arbres plans.

On note $\mathcal{A P}^{d}(n)$ le $\mathbb{Z}$-module libre sur l'ensemble des couples $(T, \phi)$, où $T$ parcourt $\operatorname{AP}^{d}(n)$ et $\phi$ est une bijection entre $\{1, \ldots, n\}$ et $\operatorname{Sect}(T)$. C'est un $\mathfrak{S}_{n}$-module libre de rang le cardinal de $\operatorname{AP}^{d}(n)$. On pose

$$
\mathcal{A} \mathcal{P}^{\bullet}(n)=\bigoplus_{d=0}^{n-1} \mathcal{A} \mathcal{P}^{d}(n) .
$$

C'est un groupe abélien libre gradué ; le degré de $(T, \phi)$ est $\operatorname{dim}(T)$.

Afin de définir la composition de l'opérade $\mathcal{A P}^{\bullet}$, on introduit les notions suivantes. Pour tout arbre $T$ et tout secteur $v \in \operatorname{Sect}(T)$, on note $\partial_{G}(v)\left(\right.$ resp. $\left.\partial_{D}(v)\right)$ l'ensemble ordonné de bas en haut des arêtes de $T$ qui bordent le secteur $v$ à gauche (resp. à droite).

D'autre part, soit $s_{\min }^{T} \in \operatorname{Som}(T)$ le sommet interne le plus bas de $T$. On note $\operatorname{Som}_{G}(T)\left(\right.$ resp. $\left.\operatorname{Som}_{D}(T)\right)$ l'ensemble ordonné de bas en haut des sommets $s \in \operatorname{Som}(T) \backslash\left\{s_{\min }^{T}\right\}$ situés sur le flanc gauche (resp. droit) de $T$, c'est à dire qui sont à l'extrêmité d'une des arêtes qui limitent l'arbre à gauche (resp. à droite).

Soient $S, T$ deux arbres plans et $v \in \operatorname{Sect}(T)$. Soit $f_{G}$ (resp. $\left.f_{D}\right)$ une application croissante au sens large $\operatorname{de}_{\operatorname{Som}_{G}}(S)\left(\operatorname{resp} . \operatorname{Som}_{D}(S)\right)$ dans $\partial_{G}(v)\left(\operatorname{resp} . \partial_{D}(v)\right)$, on note $f$ le couple $\left(f_{G}, f_{D}\right)$. Au quadruplet $(T, v, f, S)$, on associe un arbre plan $T \circ f$ défini comme suit : on place l'arbre $S$ privé de sa racine dans le secteur $v$ de sorte que le sommet $s_{\min }^{S}$ soit superposé au sommet $s(v)$ du secteur $v$ de $T$, que la courbe formée par le flanc gauche (resp. droit) de $S$ soit superposée à la courbe formée par le bord gauche (resp. droit) de $v$ et que tout sommet de $S$ appartenant à une de ces deux courbes soit placé sur l'arête qui lui est associée par $f_{G}$ ou $f_{D}$. Il existe une bijection naturelle $\operatorname{Sect}(T \circ f) \simeq \operatorname{Sect}(S) \sqcup \operatorname{Sect}(T) \backslash\{v\}$. La figure 1 

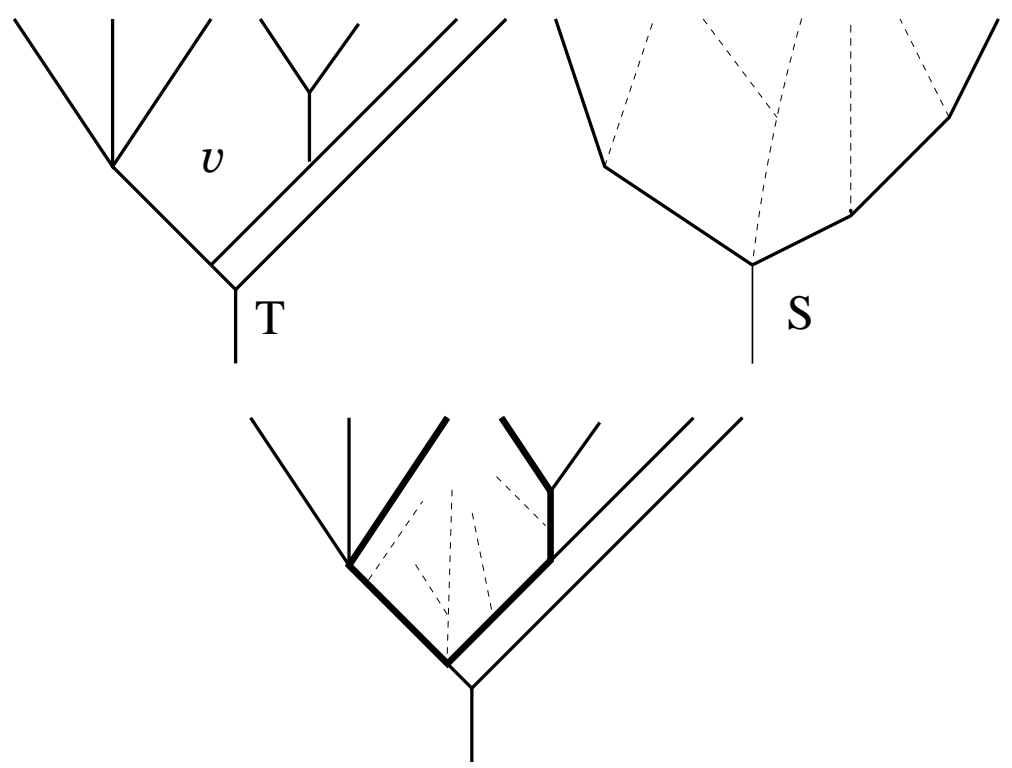

Figure 1. Exemple de terme dans la composition

montre un arbre $T$ avec un secteur distingué $v$, un arbre $S$ et un exemple d'arbre $T \circ f=v_{v} S$ pour un certain couple $f$.

Afin de simplifier la définition de la composition de l'opérade, on peut indexer les opérations de l'opérade par des ensembles finis arbitraires. On définit de façon évidente un $\mathbb{Z}$-module $\mathcal{A P}^{\bullet}(I)$ pour tout ensemble fini non vide $I$. Soient alors $I, J$ deux ensembles finis non vides, $S, T$ deux arbres plans, $\phi: I \simeq \operatorname{Sect}(S)$, $\psi: J \simeq \operatorname{Sect}(T)$ et $j \in J$. Il suffit de définir la composition de $(S, \phi)$ placé au secteur $v=\psi(j)$ de $(T, \psi)$. On pose

$$
(T, \psi) \circ_{j}(S, \phi)=\sum_{f}\left(T \circ_{v}^{f} S, \psi \circ_{j} \phi\right),
$$

où $f=\left(f_{G}, f_{D}\right), f_{G}$ (resp. $\left.f_{D}\right)$ décrit l'ensemble des applications croissantes de $\operatorname{Som}_{G}(S)\left(\operatorname{resp} . \operatorname{Som}_{D}(S)\right)$ dans $\partial_{G}(v)\left(\right.$ resp. $\left.\partial_{D}(v)\right)$ et $\psi \circ_{j} \phi$ est la bijection entre $J \backslash\{j\} \sqcup I$ et $\operatorname{Sect}(T) \backslash\{\psi(j)\} \sqcup \operatorname{Sect}(S)$ déduite de $\psi$ et $\phi$.

Proposition 1. La composition définie par (1) munit $\mathcal{A P}^{\bullet}=\left(\mathcal{A P}^{\bullet}(n)\right)_{n \geq 1}$ d'une structure d'opérade. La sous-opérade $\mathcal{A P}^{0}$ des arbres de dimension nulle est isomorphe à l'opérade Dend qui décrit les algèbres dendriformes.

Preuve. Soient $m=\# J$ et $n=\# I$, alors $\operatorname{ord}(T \circ f)=m+n-1$. Soient $d=\operatorname{dim}(S)$ et $e=\operatorname{dim}(T)$; le nombre de sommets internes de $T \circ{ }_{v}^{f} S$ est $(n-d)+(m-e)-1$, $\operatorname{donc} \operatorname{dim}(T \circ f \quad S)=d+e$. La composition respecte donc la graduation par la dimension. L'unité pour la composition est l'unique arbre plan à deux feuilles et la vérification des axiomes d'associativité et d'équivariance est immédiate. La seconde assertion de la proposition résulte de la description des algèbres dendriformes libres donnée dans [Lod99, 5.4]. 
1.3. Une opérade de groupes abéliens filtrés. On définit une opérade $\widetilde{\mathcal{A P}}$ qui est une variante filtrée de $\mathcal{A} \mathcal{P}^{\bullet}$, sur les mêmes groupes abéliens $\mathcal{A P}^{\bullet}(n)$, avec une composition modifiée qui ne respecte plus la graduation, mais respecte la filtration par les sous-groupes

$$
\mathcal{A} \mathcal{P}^{\geq d}(n)=\bigoplus_{e \geq d} \mathcal{A} \mathcal{P}^{e}(n)
$$

La composition de l'opérade $\widetilde{\mathcal{A P}}$ est définie de la façon suivante : soient $S$ et $T$ deux arbres plans et $v \in \operatorname{Sect}(T)$. On note $\widetilde{\partial_{G}}(v)$ (resp. $\left.\widetilde{\partial_{D}}(v)\right)$ l'ensemble formé par les arêtes et les sommets internes de $T(\operatorname{sauf} s(v))$ qui bordent le secteur $v$ à gauche (resp. à droite). On ordonne $\widetilde{\partial_{G}}(v)$ et $\widetilde{\partial_{D}}(v)$ de bas en haut, en plaçant chaque sommet interne entre son unique arête entrante située sur le bord et son unique arête sortante. Soient $f_{G}$ une application croissante au sens large de $\operatorname{Som}_{G}(S)$ dans $\widetilde{\partial_{G}}(v)$ et $f_{D}$ une application croissante au sens large $\operatorname{de}_{\operatorname{Som}_{D}}(S)$ dans $\widetilde{\partial_{D}}(v)$ telles que chaque sommet interne ait au plus un antécédent. On définit $T \tilde{o}_{v}^{f} S$ de façon similaire à $T \circ{ }_{v}^{f} S$, voir la figure 1 , à la différence que certains sommets internes du bord de $S$ peuvent être identifiés avec un sommet interne de $T$ au lieu d'être placés sur une arête.

Soient alors $I, J$ deux ensembles finis non vides, $S, T$ deux arbres plans, $\phi: I \simeq$ $\operatorname{Sect}(S), \psi: J \simeq \operatorname{Sect}(T)$ et $j \in J$. Il suffit de définir la composition de $(S, \phi)$ placé au secteur $v=\psi(j)$ de $(T, \psi)$. On pose

$$
(T, \psi) \tilde{\circ}_{j}(S, \phi)=\sum_{f}\left(T \tilde{\circ}_{v}^{f}, S, \psi \circ_{j} \phi\right)
$$

où $f$ est un couple $\left(f_{G}, f_{D}\right), f_{G}$ (resp. $\left.f_{D}\right)$ décrivant l'ensemble des applications croissantes au sens large de $\operatorname{Som}_{G}(S)\left(\operatorname{resp} . \operatorname{Som}_{D}(S)\right)$ dans $\widetilde{\partial_{G}}(v)\left(\operatorname{resp} . \widetilde{\partial_{D}}(v)\right)$ telles que chaque sommet interne ait au plus un antécédent et $\psi \circ_{j} \phi$ est la bijection entre $J \backslash\{j\} \sqcup I$ et $\operatorname{Sect}(T) \backslash\{\psi(j)\} \sqcup \operatorname{Sect}(S)$ déduite de $\psi$ et $\phi$.

Proposition 2. $\widetilde{\mathcal{A P}}$ est une opérade filtrée. De plus, l'opérade graduée associée gr $\widetilde{\mathcal{A P}}$ est isomorphe à $\mathcal{A} \mathcal{P}^{\bullet}$

Preuve. La démonstration des axiomes d'équivariance, d'associativité et d'unité est sans difficulté et laissée au lecteur. La seconde assertion résulte immédiatement de la définition des compositions de ces deux opérades.

Remarque. On vérifie facilement que la somme des trois arbres à trois feuilles avec l'indice 1 à gauche et le 2 à droite définit un élément associatif de $\widetilde{\mathcal{A P}}$, c'est à dire qu'il existe un (unique) morphisme d'opérades de As dans $\widetilde{\mathcal{A P}}$ qui envoie l'élément 12 qui représente le produit associatif sur cette somme d'arbres. Par conséquent, toute algèbre sur $\widetilde{\mathcal{A P}}$ est une algèbre associative.

On note $F_{\widetilde{\mathcal{A P}}} V$ la $\widetilde{\mathcal{A P}}$-algèbre libre sur un objet $V$. Elle est munie par la remarque précédente d'un produit associatif. On note $\widehat{\operatorname{Arb}}(V)$ l'algèbre associative unitaire obtenue par ajout d'une unité à $F_{\widetilde{\mathcal{A P}}} V$.

Proposition 3. Il existe un coproduit qui fait de $\widetilde{\operatorname{Arb}}(V)$ une bigèbre.

Preuve. La proposition est démontrée pour la $\widetilde{\mathcal{A P}}$-algèbre libre sur un générateur dans Cha00a, Prop. 2]. Le coproduit est donné dans ce cas par une formule purement combinatoire, en termes d'arbres plans. 
Soit $\Gamma$ une base de $V$, alors $\widetilde{\operatorname{Arb}}(V)$ admet une base formée par les arbres plans décorés par $\Gamma$. La formule pour le coproduit donnée dans Cha00a Form. (6)] s'étend de façon naturelle au cas des arbres décorés. On vérifie alors de manière exactement analogue, comme dans [Cha00a], la compatibilité avec le produit.

1.4. Une opérade dans la catégorie des complexes. Soit $T$ un arbre plan. On appelle arêtes extrêmes d'un sommet $s \in \operatorname{Som}(T)$ les arêtes entrantes en $s$ situées le plus à gauche et le plus à droite. On note $\operatorname{Nex}(s)$ l'ensemble des arêtes non-extrêmes du sommet $s \in \operatorname{Som}(T)$ et $\operatorname{Nex}(T)=\bigsqcup_{s} \operatorname{Nex}(s)$. On vérifie que le cardinal de $\operatorname{Nex}(T)$ est égal à $\operatorname{dim}(T)$. On note $\operatorname{dim}(s)$ le cardinal de $\operatorname{Nex}(s)$. On appelle orientation de l'arbre $T$ la donnée d'une orientation or de l'espace vectoriel $\mathbb{R} \operatorname{Nex}(T)$, c.-à-d. la donnée d'un produit extérieur des vecteurs correspondant aux éléments de $\operatorname{Nex}(T)$. On définit $\omega_{s}$ comme le produit extérieur de gauche à droite des vecteurs de $\mathbb{R} \operatorname{Nex}(T)$ correspondant aux éléments de $\operatorname{Nex}(s)$.

On note $\operatorname{AP}_{D G}^{d}(n)$ l'ensemble des arbres plans d'ordre $n$ et de dimension $d$ munis d'une orientation au sens ci-dessus. Il existe une bijection naturelle entre $\bigsqcup_{d} \operatorname{AP}_{D G}^{d}(n)$ et l'ensemble des faces orientées du polytope de Stasheff de dimension $n-1$, qui envoie les arbres de dimension $d$ sur les faces de dimension $d$, voir Cha00b 4.1] pour plus de détails.

On va définir une opérade $\mathcal{A} \mathcal{P}_{D G}$ dans la catégorie monoïdale symétrique $\mathrm{Ab}_{D G}$ des complexes de groupes abéliens, munie du produit tensoriel usuel et de la volte $\tau$ définie par $\tau(u \otimes v)=(-1)^{|u||v|} v \otimes u$, où $|u|$ désigne le degré de $u$.

Soient $n \geq 1$ et $0 \leq d \leq n-1$. On note $\mathcal{A P}_{D G}^{d}(n)$ le quotient du $\mathbb{Z}$-module libre sur les triplets $(T, o r, \phi)$ où $T$ est un arbre plan d'ordre $n$ et de dimension $d$, or une orientation de $T$ au sens ci-dessus et $\phi$ une bijection de $\{1, \ldots, n\} \operatorname{dans} \operatorname{Sect}(T)$, par les relations $(T,-o r, \phi)=-(T, o r, \phi)$, et on pose

$$
\mathcal{A P}_{D G}^{\bullet}(n)=\bigoplus_{d=0}^{n-1} \mathcal{A} \mathcal{P}_{D G}^{d}(n)
$$

Pour définir la différentielle $\delta$ du complexe $\mathcal{A P}_{D G}^{\bullet}(n)$, on aura besoin de quelques notations. Soit $T$ un arbre plan. Une arête $a$ de $T$ est dite interne si ses deux extrêmités sont des sommets internes de $T$. On note alors $s_{a}^{-}$le sommet initial et $s_{a}^{+}$le sommet final de $a$. On note $\operatorname{Int}(T)$ l'ensemble des arêtes internes de $T$. Si $a \in \operatorname{Int}(T)$, on note $D_{a}(T)$ l'arbre obtenu par contraction de $a$ et on désigne par $s_{a}$ le sommet de $D_{a}(T)$ correspondant à la fusion de $s_{a}^{-}$et $s_{a}^{+}$. On observe qu'il y a une bijection naturelle entre $\operatorname{Sect}(T)$ et $\operatorname{Sect}\left(D_{a}(T)\right)$. Soit $a \in \operatorname{Int}(T)$, on note $\operatorname{ord}_{G}(a)$ le nombre de secteurs de $s_{a}^{+}$à gauche de l'arête $a$.

On peut maintenant définir la différentielle $\delta$. Si $d=n-1$, on pose $\delta(T$, or, $\phi)=$ 0 . Pour $0 \leq d \leq n-2$, on pose

$$
\delta(T, \text { or }, \phi)=\sum_{a \in \operatorname{Int}(T)}(-1)^{\operatorname{ord}\left(s_{a}^{-}\right) \operatorname{ord}_{G}(a)}\left(D_{a}(T), \Omega_{a} \wedge \text { or }, \phi\right),
$$

où $\Omega_{a}$ est un symbole de degré un tel que $\omega_{s_{a}^{-}} \wedge \Omega_{a} \wedge \omega_{s_{a}^{+}}=\omega_{s_{a}}$. Il résulte de (Cha00b 4.3]) que $\delta$ est bien une différentielle. Les complexes ainsi obtenus sont les complexes duaux des complexes cellulaires des polytopes de Stasheff.

Il reste à définir la composition. Soient $(T, v, f, S)$ comme dans la formule (1), alors on a une bijection naturelle $\operatorname{Nex}(T \circ f) \Omega \operatorname{Nex}(T) \sqcup \operatorname{Nex}(S)$. Si $T$ est orienté par $0 r_{T}$ et $S$ par $o r_{S}$, on peut alors orienter $T \circ{ }_{v}^{f} S$ par $o r_{T} \wedge o r_{S}$. Si $v=\psi(j)$, on 
pose

$$
\left(T, o r_{T}, \psi\right) \circ_{j}\left(S, o r_{S}, \phi\right)=\sum_{f}\left(T \circ f{ }_{v}^{f} S, o r_{T} \wedge o r_{S}, \psi \circ_{j} \phi\right),
$$

où $f$ est un couple $\left(f_{G}, f_{D}\right), f_{G}$ décrit les applications croissantes de $\operatorname{Som}_{G}(S)$ dans $\partial_{G}(v)$ et $f_{D}$ décrit les applications croissantes de $\operatorname{Som}_{D}(S)$ dans $\partial_{D}(v)$.

Le seul point délicat dans la vérification des axiomes est l'objet du lemme suivant.

Lemme 1. La composition ainsi définie est un morphisme de complexes de $\mathcal{A P}_{D G}^{\bullet}(J) \otimes \mathcal{A P}_{D G}^{\bullet}(I)$ dans $\mathcal{A P}_{D G}^{\bullet}(J \backslash\{j\} \sqcup I)$.

Preuve. Pour tout couple $f=\left(f_{G}, f_{D}\right)$, on observe qu'il y a une bijection évidente

$$
\operatorname{Som}\left(T \circ{ }_{v}^{f} S\right) \simeq \operatorname{Som}(T) \backslash\{s(v)\} \sqcup \operatorname{Som}(S) \backslash\left\{s_{\min }^{S}\right\} \sqcup\left\{s_{\text {NOV }}\right\},
$$

où $s_{\text {NOV }}$ désigne le sommet où se superposent $s(v)$ et $s_{\text {min }}^{S}$. Dans la suite, on identifie tous ces ensembles par ces bijections.

Par définition, $\delta\left(\left(T, o_{T}, \psi\right) \circ_{j}\left(S, o r_{S}, \phi\right)\right)$ est égal à

$$
\sum_{f} \sum_{a \in \operatorname{Int}\left(T \circ_{v}^{f} S\right)}(-1)^{\operatorname{ord}\left(s_{a}^{-}\right) \operatorname{ord}_{G}(a)}\left(D_{a}(T \circ f), \Omega_{a} \wedge \text { or }_{T} \wedge \text { or }_{S}, \psi \circ_{j} \phi\right) .
$$

On découpe cette somme en trois termes selon que l'on a

1. $s_{a}^{+}$et $s_{a}^{-} \in \operatorname{Som}(S) \backslash\left\{s_{\min }^{S}\right\} \sqcup\left\{s_{\mathrm{NOV}}\right\}$,

2. $s_{a}^{+}$et $s_{a}^{-} \in \operatorname{Som}(T) \backslash\{s(v)\} \sqcup\left\{s_{\text {NOV }}\right\}$,

3 . les autres cas.

Le terme correspondant au cas 1 . se réécrit

$$
\sum_{a \in \operatorname{Int}(S)} \sum_{f^{\prime}}(-1)^{\operatorname{ord}\left(s_{a}^{-}\right) \operatorname{ord}_{G}(a)}\left(T \circ{ }_{v}^{f^{\prime}} D_{a}(S),(-1)^{\operatorname{dim}(T)} \operatorname{or}_{T} \wedge \Omega_{a} \wedge o r_{S}, \psi \circ_{j} \phi\right),
$$

où $f^{\prime}$ est un couple $\left(f_{G}^{\prime}, f_{D}^{\prime}\right), f_{G}^{\prime}$ une application croissante de $\operatorname{Som}_{G}\left(D_{a}(S)\right)$ dans $\partial_{G}(v)$ et $f_{D}^{\prime}$ une application croissante de $\operatorname{Som}_{D}\left(D_{a}(S)\right)$ dans $\partial_{D}(v)$. Ce premier terme est donc égal à

$$
(-1)^{\operatorname{dim}(T)}\left(T, o r_{T}, \psi\right) \circ_{j} \delta\left(S, o r_{S}, \phi\right) .
$$

Le terme correspondant au cas 2 . se réécrit de manière similaire, en distinguant selon que $a$ appartient à $\partial_{G}(v) \sqcup \partial_{D}(v)$ ou non. On montre de la sorte que ce terme est égal à

$$
\sum_{a \in \operatorname{Int}(T)} \sum_{f^{\prime}}(-1)^{\operatorname{ord}\left(s_{a}^{-}\right) \operatorname{ord}_{G}(a)}\left(D_{a}(T) \circ f_{v}^{\prime} S, \Omega_{a} \wedge \text { or }_{T} \wedge \text { or }_{S}, \psi \circ_{j} \phi\right),
$$

où $f^{\prime}$ est un couple $\left(f_{G}^{\prime}, f_{D}^{\prime}\right), f_{G}^{\prime}$ une application croissante $\operatorname{de}_{\operatorname{Som}_{G}}(S)$ dans $\partial_{G}(v)$ et $f_{D}^{\prime}$ une application croissante $\operatorname{de} \operatorname{Som}_{D}(S)$ dans $\partial_{D}(v)$. Ce second terme est donc égal à

$$
\delta\left(T, o r_{T}, \psi\right) \circ_{j}\left(S, o r_{S}, \phi\right) .
$$

Montrons que le terme correspondant au cas 3 est nul. Nécessairement, on a $a \in \partial_{G}(v) \sqcup \partial_{D}(v)$. Montrons que le terme correspondant à $a \in \partial_{G}(v)$ est nul, le cas $a \in \partial_{D}(v)$ étant similaire.

Soit $a \in \partial_{G}(v)$. On distingue deux cas :

$$
\left\{\begin{array}{l}
s_{a}^{-} \in \operatorname{Som}_{G}(S) \text { et } s_{a}^{+} \in \operatorname{Som}(T) \backslash\{s(v)\} \\
s_{a}^{+} \in \operatorname{Som}_{G}(S) \text { et } s_{a}^{-} \in \operatorname{Som}(T) \backslash\{s(v)\}
\end{array}\right.
$$


On définit une bijection entre les deux parties de l'ensemble de sommation correspondant à ces deux cas. A un couple $(f, a)$ avec

$$
s_{a}^{-} \in \operatorname{Som}_{G}(S) \text { et } s_{a}^{+} \in \operatorname{Som}(T) \backslash\{s(v)\},
$$

on associe le couple $\left(f^{\prime}, a^{\prime}\right)$ obtenu comme suit : le couple $f^{\prime}=\left(f_{G}^{\prime}, f_{D}^{\prime}\right)$ est défini ainsi : $f_{D}^{\prime}=f_{D}$, et $f_{G}^{\prime}$ coïncide avec $f_{G}$ sur $\operatorname{Som}_{G}(S)$, sauf en le sommet $s_{a}^{-}$de $S$, où $f_{G}^{\prime}\left(s_{a}^{-}\right)$est l'arête sortante du sommet $s_{a}^{+}$de $T$. L'arête $a^{\prime}$ est l'unique arête de $T \circ{ }_{v}^{f^{\prime}} S$ de sommet initial $s_{a}^{+}$et de sommet final $s_{a}^{-}$. Dans cette situation, $T \circ f$ et $T \circ f_{v}^{f^{\prime}} S$ ne diffèrent que par un changement local au voisinage des sommets $s_{a}^{-}$ et $s_{a}^{+}$, voir les configurations de gauche et de droite de la figure 2 .

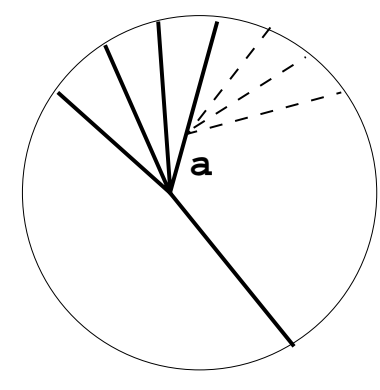

$(f, a)$
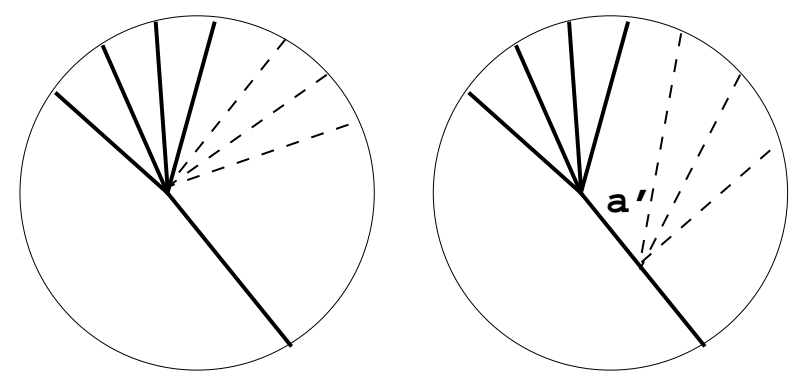

$\left(f^{\prime}, a^{\prime}\right)$

Figure 2. Configurations locales

On a alors $\left.D_{a}(T \circ f) S\right)=D_{a^{\prime}}\left(T \circ f_{v}^{f^{\prime}} S\right)$, c'est l'arbre correspondant à la configuration du milieu de la figure 2 .

On va montrer que les deux termes correspondant à $(f, a)$ et $\left(f^{\prime}, a^{\prime}\right)$ sont opposés. Comme $\operatorname{ord}_{G}(a)=\operatorname{ord}\left(s_{a}^{+}\right)$, le terme associé à $(f, a)$ est

$$
(-1)^{\operatorname{ord}\left(s_{a}^{-}\right) \operatorname{ord}\left(s_{a}^{+}\right)}\left(D_{a}\left(T \circ_{v}^{f} S\right), \Omega_{a} \wedge \operatorname{or}_{T} \wedge o r_{S}, \psi \circ_{j} \phi\right) .
$$

Comme $\operatorname{ord}_{G}\left(a^{\prime}\right)=0$, le terme associé à $\left(f^{\prime}, a^{\prime}\right)$ est

$$
\left(D_{a^{\prime}}\left(T \circ_{v}^{f^{\prime}} S\right), \Omega_{a^{\prime}} \wedge o r_{T} \wedge o r_{S}, \psi \circ_{j} \phi\right) .
$$

Il s'agit donc de montrer que les orientations $(-1)^{\operatorname{ord}\left(s_{a}^{-}\right)} \operatorname{ord}\left(s_{a}^{+}\right) \Omega_{a} \wedge \operatorname{or}_{T} \wedge$ or $r_{S}$ et $\Omega_{a^{\prime}} \wedge$ or $_{T} \wedge$ or $_{S}$ sont opposées. Le sommet initial de $a^{\prime}$ correspond au sommet $s_{a}^{+}$ de $S$, son sommet final au sommet $s_{a}^{-}$. Par définition des symboles $\Omega$, on a

$$
\omega_{s_{a}^{-}} \wedge \Omega_{a} \wedge \omega_{s_{a}^{+}}=\omega_{s_{a}^{+}} \wedge \Omega_{a^{\prime}} \wedge \omega_{s_{a}^{-}},
$$

donc

$$
\Omega_{a} \wedge \omega_{s_{a}^{-}} \wedge \omega_{s_{a}^{+}}=(-1)^{\operatorname{dim}\left(s_{a}^{+}\right) \operatorname{dim}\left(s_{a}^{-}\right)+\operatorname{dim}\left(s_{a}^{+}\right)+\operatorname{dim}\left(s_{a}^{-}\right)} \Omega_{a^{\prime}} \wedge \omega_{s_{a}^{-}} \wedge \omega_{s_{a}^{+}} .
$$

Le fait que, pour tout arbre $T$ et pour tout $s \in \operatorname{Som}(T)$, on ait $\operatorname{ord}(s)=\operatorname{dim}(s)+1$ permet de conclure que les signes sont opposés. Par conséquent, la somme des termes dans le cas 3 est nulle. Ceci termine la démonstration du lemme.

Proposition 4. La composition définie par (5) munit $\mathcal{A P}_{D G}=\left(\mathcal{A P}_{D G}^{\bullet}(n)\right)_{n \geq 1}$ d'une structure d'opérade différentielle graduée. 
Preuve. La démonstration des axiomes d'équivariance, d'associativité et d'unité est sans difficulté et laissée au lecteur.

Remarque. On vérifie facilement que la somme des deux arbres à trois feuilles de dimension nulle avec l'indice 1 à gauche et le 2 à droite définit un élément associatif fermé de $\mathcal{A P}_{D G}$, c'est à dire qu'il existe un (unique) morphisme d'opérades différentielles graduées de As dans $\mathcal{A P}_{D G}$ qui envoie l'élément 12 qui représente le produit associatif sur cette somme d'arbres. Ce morphisme est un quasiisomorphisme, c'est à dire qu'il induit un isomorphisme en homologie. Par conséquent, toute algèbre sur $\mathcal{A P}_{D G}$ est une algèbre associative.

On note $F_{\mathcal{A} \mathcal{P}_{D G}} V$ la $\mathcal{A} \mathcal{P}_{D G}$-algèbre libre sur un objet $V$. Elle est munie par la remarque précédente d'un produit associatif. On note $\operatorname{Arb}_{D G}(V)$ l'algèbre associative unitaire obtenue par ajout d'une unité à $F_{\mathcal{A} \mathcal{P}_{D G}} V$.

Proposition 5. Il existe un coproduit qui fait de $\operatorname{Arb}_{D G}(V)$ une bigèbre différentielle graduée.

Preuve. La proposition est démontrée pour la $\mathcal{A} \mathcal{P}_{D G}$-algèbre libre sur un générateur dans Cha00b, Thm. 2]. Le coproduit est donné dans ce cas par une formule purement combinatoire, en termes d'arbres plans.

Soit $\Gamma$ une base de $V$, alors $\operatorname{Arb}_{D G}(V)$ admet une base indexée par les arbres plans décorés par $\Gamma$. La formule pour le coproduit donnée dans Cha00b 4.5] s'étend de façon naturelle au cas des arbres décorés. On vérifie alors de manière exactement analogue, comme dans [Cha00b], la compatibilité avec le produit.

\section{Opérades Sur les faces Des hypercubes}

2.1. Généralités sur les hypercubes. Soient $n \geq 1$ et $0 \leq d \leq n-1$, on appelle montagne d'ordre $n$ et de dimension $d$ un $(n-1)$-uplet d'éléments de $\{-1,0,+1\}$ comportant exactement $d$ zéros. On représente une montagne par une ligne brisée de $n-1$ segments dont la direction est NO-SE, O-E ou SO-NE selon que le coefficient est $+1,0$ ou -1 . On appelle montagne numérotée la donnée d'une montagne d'ordre $n$ et d'une indexation par l'ensemble $\{1, \ldots, n\}$ des extrémités des segments (voir Fig. 3).

Soit $\Gamma$ un ensemble fini. On appelle montagne décorée par $\Gamma$ un couple $(C, \gamma)$, où $C$ est une montagne et $\gamma$ une application de l'ensemble des extrémités des segments de $C$ dans $\Gamma$.

2.2. Une opérade de groupes abéliens gradués. On va définir une opérade $\mathcal{M N}$ dans la catégorie monoïdale symétrique $\mathrm{Ab}_{g r}$. Soit $\operatorname{MN}^{d}(n)$ l'ensemble des montagnes numerotées d'ordre $n$ et de dimension $d$. On note $\mathcal{M N}^{d}(n)$ le $\mathbb{Z}$-module

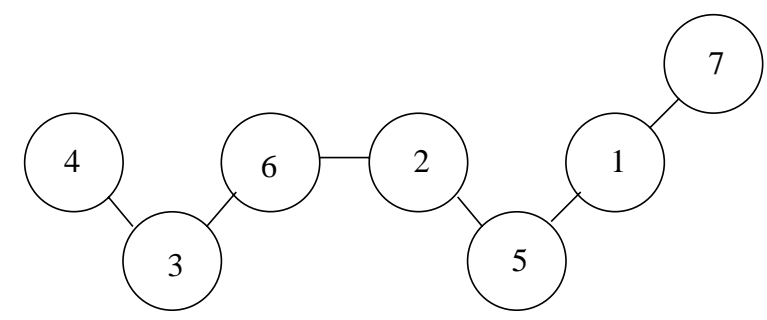

Figure 3. Exemple de montagne numérotée : $(+1,-1,0,+1,-1,-1)$ 
libre sur $\operatorname{MN}^{d}(n)$ et $\mathcal{M N}^{\bullet}(n)=\bigoplus_{d=0}^{n-1} \mathcal{M} \mathcal{N}^{d}(n)$. Par commodité, on étend comme précédemment ces définitions aux ensembles finis. Soient alors $I, J$ deux ensembles finis, $B \in \mathrm{MN}(I), C \in \mathrm{MN}(J)$ et $j \in J$. On définit $C \circ_{j} B$ comme la montagne numérotée obtenue en remplaçant dans $C$ le point $j$ par la montagne $B$ (voir Fig. 4.).

Proposition 6. La composition ci-dessus définit une structure d'opérade sur $\mathcal{M N}$ $=\left(\mathcal{M N}^{\bullet}(n)\right)_{n \geq 1}$.

Preuve. La composition respecte la graduation par la dimension. La démonstration des axiomes d'équivariance, d'associativité et d'unité est sans difficulté et laissée au lecteur.

Remarque. On vérifie facilement que $(-1)+(0)+(+1)$ avec l'indice 1 à gauche et le 2 à droite définit un élément associatif de $\mathcal{M N}$, c'est à dire qu'il existe un (unique) morphisme d'opérades de As dans $\mathcal{M N}$ qui envoie l'élément 12 qui représente le produit associatif sur cette somme de montagnes. Par conséquent, toute algèbre sur $\mathcal{M N}$ est une algèbre associative.

On note $F_{\mathcal{M N}} V$ la $\mathcal{M N}$-algèbre libre sur un objet $V$ dans $\mathrm{Ab}_{g r}$. Elle est munie par la remarque précédente d'un produit associatif. On note $\mathrm{Cub}(V)$ l'algèbre associative unitaire obtenue par ajout d'une unité à $F_{\mathcal{M N}} V$.

Proposition 7. Il existe un coproduit qui fait de $\mathrm{Cub}(V)$ une bigèbre.

Preuve. La proposition est démontrée pour la $\mathcal{M N}$-algèbre libre sur un générateur dans Cha00a Prop. 3]. Le coproduit est donné dans ce cas par une formule purement combinatoire, en termes de montagnes.

Soit $\Gamma$ une base de $V$, alors $\operatorname{Cub}(V)$ admet une base indexée par les montagnes décorés par $\Gamma$. La formule pour le coproduit donnée dans [Cha00a, Lem. 5] s'étend de façon naturelle au cas des montagnes décorées. On vérifie alors de manière exactement analogue, comme dans Cha00a, la compatibilité avec le produit.

2.3. Une opérade dans la catégorie des complexes. Soit $B$ une montagne, c.-à-d. un $(n-1)$-uplet de coefficients $\{-1,0,+1\}$. On note $\operatorname{Zer}(B)$ l'ensemble des coefficients nuls de $B$; le cardinal de $\operatorname{Zer}(B)$ est donc égal à $\operatorname{dim}(B)$. On appelle orientation de $B$ la donnée d'une orientation or de l'espace vectoriel $\mathbb{R} \operatorname{Zer}(B)$ c.à-d. la donnée d'un produit extérieur des vecteurs correspondant aux éléments de $\operatorname{Zer}(B)$. On note $\mathrm{MN}_{D G}^{d}(n)$ l'ensemble des montagnes numérotées d'ordre $n$ et de dimension $d$ munies d'une orientation au sens ci-dessus. On observe qu'il y a une bijection naturelle entre les montagnes orientées d'ordre $n$ et de dimension $d$ et les faces orientées de dimension $d$ de l'hypercube de dimension $n-1$.

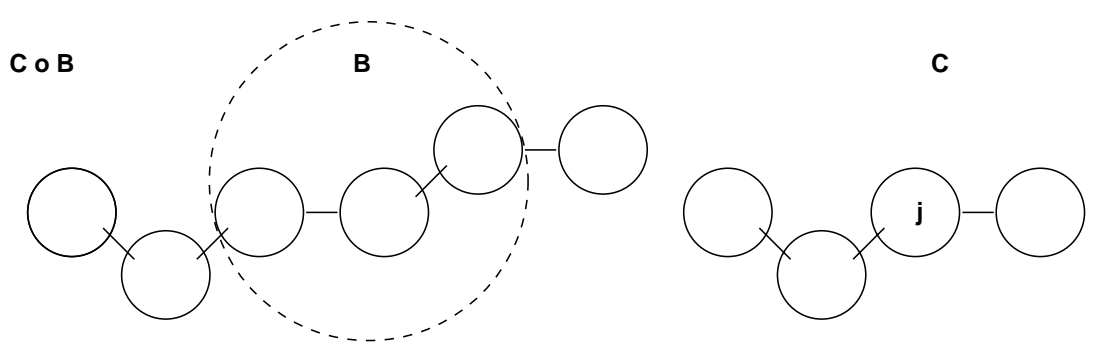

Figure 4. Composition de montagnes : $C \circ_{j} B$ 
On va définir une opérade $\mathcal{M} \mathcal{N}_{D G}$ dans la catégorie $\mathrm{Ab}_{D G}$. Pour $n \geq 1$ et $0 \leq d \leq n-1$, on note $\mathcal{M} \mathcal{N}_{D G}^{d}(n)$ le quotient du $\mathbb{Z}$-module libre sur les couples $(B$, or $) \in \operatorname{MN}_{D G}^{d}(n)$ par les relations $(B,-o r)=-(B, o r)$, et on pose

$$
\mathcal{M N}_{D G}^{\bullet}(n)=\bigoplus_{d=0}^{n-1} \mathcal{M} \mathcal{N}_{D G}^{d}(n)
$$

On définit la différentielle du complexe $\mathcal{M N}_{D G}^{\bullet}(n)$ comme suit :

$$
\delta(B, \text { or })=\sum_{i=1}^{n-1} B(i)\left(D_{i}(B), i \wedge \text { or }\right),
$$

où $B(i)$ désigne le $i^{\text {ème }}$ coefficient de $B$ et où $D_{i}(B)$ est défini par

$$
\left\{\begin{array}{l}
D_{i}(B)(j)=B(j) \text { si } j \neq i, \\
D_{i}(B)(i)=0 .
\end{array}\right.
$$

Soient $I, J$ et $B, C$ comme plus haut, alors on a une bijection naturelle $\operatorname{Zer}\left(C \circ_{j} B\right) \simeq$ $\operatorname{Zer}(B) \sqcup \operatorname{Zer}(C)$. Si $B$ est orienté par $o_{B}$ et $C$ par or $C$, on peut alors orienter $C \circ_{j} B$ par or $_{C} \wedge$ or $_{B}$.

Proposition 8. La composition définie par

$$
\left(C, \text { or }_{C}\right) \circ_{j}\left(B, o r_{B}\right)=\left(C \circ_{j} B, o r_{C} \wedge o r_{B}\right)
$$

munit $\mathcal{M N}_{D G}=\left(\mathcal{M N}_{D G}^{\bullet}(n)\right)_{n \geq 1}$ d'une structure d'opérade.

Preuve. La vérification que la composition est un morphisme de complexe et celle des axiomes d'associativité, d'équivariance et d'unité est immédiate.

Remarque. On vérifie facilement que $(-1)+(+1)$ avec l'indice 1 à gauche et le 2 à droite définit un élément associatif fermé de $\mathcal{M} \mathcal{N}_{D G}$, c'est à dire qu'il existe un (unique) morphisme d'opérades différentielles graduées de As dans $\mathcal{M} \mathcal{N}_{D G}$ qui envoie l'élément 12 qui représente le produit associatif sur cette somme de montagnes. Ce morphisme est un quasi-isomorphisme, c'est à dire qu'il induit un isomorphisme en homologie. Par conséquent, toute algèbre sur $\mathcal{M N}_{D G}$ est une algèbre associative.

Soit $F_{\mathcal{M N}_{D G}} V$ la $\mathcal{M} \mathcal{N}_{D G}$-algèbre libre sur un objet $V$ dans $\mathrm{Ab}_{D G}$. Elle est munie par la remarque précédente d'un produit associatif. On note $\operatorname{Cub}_{D G}(V)$ l'algèbre associative unitaire obtenue par ajout d'une unité à $F_{\mathcal{M N}_{D G}} V$.

Proposition 9. Il existe un coproduit qui fait de $\operatorname{Cub}_{D G}(V)$ une bigèbre différentielle graduée.

Preuve. La proposition est démontrée pour la $\mathcal{M N} \mathcal{N}_{D G}$-algèbre libre sur un générateur dans [Cha00b, Thm. 3].

Soit $\Gamma$ une base de $V$, alors $\operatorname{Cub}_{D G}(V)$ admet une base indexée par les montagnes décorées par $\Gamma$.

La construction du coproduit donnée dans Cha00b, 5] s'étend de façon naturelle au cas des montagnes décorées. On vérifie alors de manière exactement analogue, comme dans Cha00b, la compatibilité avec le produit. 


\section{REFERENCES}

[Cha00a] Frédéric Chapoton, Algèbres de Hopf des permutohèdres, associahèdres et hypercubes, Adv. Math. 150 (2000), no. 2, 264-275. MR 2001c:16066

[Cha00b] Frédéric Chapoton, Bigèbres différentielles graduées associées aux permutoèdres, associaèdres et hypercubes, Annales de l'Institut Fourier 50 (2000), 1127-1153.

[CK98] Alain Connes and Dirk Kreimer, Hopf algebras, renormalization and noncommutative geometry, Comm. Math. Phys. 199 (1998), no. 1, 203-242. MR 99h:81137

[KS00] Maxim Kontsevich and Yan Soibelman, Deformations of algebras over operads and Deligne's conjecture, preprint QA/0001151, january 2000.

[Lod95] Jean-Louis Loday, Algèbres ayant deux opérations associatives (digèbres), C. R. Acad. Sci. Paris Sér. I Math. 321 (1995), no. 2, 141-146. MR 96f:16013

[Lod97] - Overview on Leibniz algebras, dialgebras and their homology, Cyclic cohomology and noncommutative geometry (Waterloo, ON, 1995), Amer. Math. Soc., Providence, RI, 1997, pp. 91-102. MR 98j:17001

[Lod99] - Dialgebras, Prépublication de l'IRMA (Strasbourg) Vol 14, 1999.

[LR98] Jean-Louis Loday and Maria O. Ronco, Hopf algebra of the planar binary trees, Advances in Math. 139 (1998), no. 2, 293-309. MR 99m:16063

[MR95] Claudia Malvenuto and Christophe Reutenauer, Duality between quasi-symmetric functions and the Solomon descent algebra, J. Algebra 177 (1995), no. 3, 967-982. MR 97d:05277

[Ron00] María Ronco, Primitive elements in a free dendriform algebra. New trends in Hopf algebra theory (La Falda, 1999), Contemporary Math. 267, A.M.S., Providence, RI, 2000 , pp. $245-253$.

[Sta63] James Dillon Stasheff, Homotopy associativity of H-spaces. I, II, Trans. Amer. Math. Soc. 108 (1963), 275-292; ibid. 108 (1963), 293-312. MR 28:1623

Equipe Analyse Algébrique, Case 82, Institut de Mathématiques, 175 Rue du Chevaleret 75013 Paris, France

E-mail address: chapoton@math.jussieu.fr 